April 24th, 1994

\title{
Regge Asymptotics of Scattering with Flavour Exchange in QCD
}

\author{
R. Kirschner \\ DESY - Institut für Hochenergiephysik, Zeuthen, Germany
}

\begin{abstract}
The contribution to the perturbative Regge asymptotics of the exchange of two reggeized fermions with opposite helicity is investigated. The methods of conformal symmetry known for the case of gluon exchange are extended to this case where double-logarithmic contributions dominate the asymptotics. The Regge trajectories at large momentum transfer are calculated.
\end{abstract}




\section{Introduction}

The phenomenology of processes dominated by the perturbative Regge kinematics, the overlap of the Regge region and the range of applicability of perturbative QCD, attracts increasing interest. The leading singularity of the vacuum channel in perturbative QCD, the perturbative pomeron, is well investigated [1] [2]. It is expected to show up in the low $x$ asymptotics of structure functions [3], in jet-inclusive final states with rapidity gaps in deep-inelastic, hadron-hadron and other processes [4]. More effort has to be applied to work out the unitarity corrections to the leading logarithmic approximation in order to calculate the true asymptotics [5] [6] [7] [8] [9] [10].

The perturbative Regge singularities of the channels with quantum number exchange, carried by quark and anti-quark of different flavour, dominate in more specific quantities, which are more difficult to measure in the kinematical range of interest with enough accuracy. They should show up in the small $x$ behaviour of flavour non-singlet structure functions, corresponding distributions with jets at rapidity gaps and in the diffractive hadronic scattering with relatively large momentum transfer and identified particles [11].

The perturbative Regge asymptotics of these channels has been calculated in the double logarithmic approximation [12] [13]. The partial-wave equation in the singlelogarithmic approximation has been considered in [14] [15].

The double-logarithmic contributions prevented a straightforward extension of the methods developed for the perturbative pomeron [2] [8] [9] [16], where such contributions do not appear. The pomeron equation transformed into the impact parameter space shows conformal symmetry and holomorphic factorization. These properties are very useful in treating the multi reggeon exchange, which in the case of only gluons gives the desired unitarity corrections to the perturbative pomeron and the odderon [17].

It is important to extend the ideas developed for gluon exchange to fermions. A treatment of multi-reggeon exchange including fermions is needed for a complete understanding of the Regge asymptotics in perturbative QCD. The generalization of the methods to fermions gives a much deeper theoretical understanding. The generalization of the effective action approach [7] serves as a good example.

In the present paper we show that the properties of conformal symmetry and holomorphic factorization hold also for the two-fermion exchange. The double-logarithmic contributions are no obstacle any more for aplying the methods developed in [2] [8] [9] [16]. Here we restrict ourselves to the case of opposite helicities of the exchanged fermions, because the double logarithms arise in this case only.

The scalar $\phi^{3}$ in 6 dimensions served as a toy model providing an understanding of how conformal symmetry may be compatible with double logarithms [18]. The operator representation [8] [9] gave the appropriate framework for solving our problem. The approach developed in [2] [19] has been applied for improving the fixed coupling approximation. The quark anti-quark Regge trajectories have been studied in the large $N_{C}$ approximation in [20].

We rederive the partial-wave equation starting from the effective graphical rules on which the effective action is based [7]. We solve the equation first at vanishing momentum 
transfer, find the leading Regge singularities and show, that the eigenvalue spectrum is compatible with conformal symmetry and holomorphic factorization. Then the operator representation in the impact parameter space is investigated. We generalize the conformal approach to the case where the exchanged particles correspond to conformal operators with non-vanishing conformal weight. We derive a representation of the equation, where the operator involved is a sum of a function of the Casimir operator of the holomorphic conformal transformations and a function of the corresponding anti-holomorphic Casimir operator. Finally we calculate the series of moving Regge poles emerging from the fixed branch-points by imposing the condition of continuity at the transition from the Regge region to the deep inelastic region, where renormalization group determines the behaviour.

\section{The partial-wave equation}

We present a short derivation of the partial-wave equation starting from the effective graphical rules [7]. The bremsstrahlung contributions are already included in the effective vertex and in the fermion trajectory. Therefore a discussion of bremsstrahlung separation as in another derivation [15] is not needed here.

In the logarithmic approximation the leading contribution arises from the t-channel exchange of two reggeized fermions, which interact by exchanging s-channel gluons. The effective vertex of gluon emission by an exchanged fermion is given by (Fig. 1a)

$$
T_{\alpha \alpha^{\prime}}^{a}\left(\phi^{* a} \kappa^{\prime *}+\phi^{a} \kappa^{\prime *}\right)
$$

$k$ is the four-momentum and $\kappa$ its transverse (with respect to the momenta of the incoming particles) part in complex notation $\left(\kappa=\kappa_{1}+i \kappa_{2}\right) . \phi^{a}$ and its complex conjugate stand for the wave functions of the gluon of helicity -1 or +1 , respectively. The helicity state of the exchanged fermion is indicated by an arrow on the line in Fig. 1; the vertex and the propagator corresponding to the opposite helicity are obtained by complex conjugation. The fermion propagator (Fig. 1b) is given by

$$
\frac{1}{\kappa^{*}}
$$

and that of the s-channel gluon by (Fig. 1b)

$$
\left\langle\phi^{*} \phi\right\rangle=\frac{1}{\left(k-k^{\prime}\right)^{2}+i \epsilon} \frac{1}{\mid\left(\kappa-\left.\kappa^{\prime}\right|^{2}\right.}, \quad\langle\phi \phi\rangle=0 .
$$

In the fermion propagator the condition of multi-Regge kinematics that the longitudinal part $k_{+} k_{-}$of the momentum squared $k^{2}=k_{+} k_{-}-|\kappa|^{2}$ is small compared to the transverse part has to be imposed, maybe by a step function multiplying (2.2). (Compared to [7] in the expressions (2.1)-(2.3) we changed consistently some factors of 2 ).

The trajectory of the reggeized fermion is $\frac{1}{2}+\alpha_{F}(\kappa)$, where

$$
\alpha_{F}(\kappa)=-\frac{g^{2} C_{2}}{(2 \pi)^{3}} \int \frac{d^{2} \kappa^{\prime}}{\left|\kappa-\kappa^{\prime}\right|^{2}} \frac{\kappa}{\kappa^{\prime}}
$$


Here a regularization has to be adopted, e.g. by extending the dimension of the transverse space $2 \rightarrow 2+2 \epsilon$. In this regularization the trajectory does not depend on the helicity.

The amplitude of the flavour-exchange high-energy scattering has the structure given by the graph Fig. 2. We shall not be concerned with the impact factors $\Phi$ depending on the scattering particles. We concentrate on the universal two-reggeon Green function, describing the exchange two reggeized fermions of opposite helicity inteacting by gluon exchange. It obeys the equation represented graphically in Fig. 3.

We have collected the ingredients for writing the equation. The propagator of the two reggeized fermions (the first term on r.h.s.) is given by

$$
\delta^{(2)}(\kappa-\bar{\kappa})\left[\kappa^{*}(q-\kappa)\left(\omega-\alpha_{F}(\kappa)-\alpha_{F}(q-\kappa)\right)\right]^{-1}
$$

For the amplitude Fig. 2 we use the Mellin representation with $\omega$ being the angular momentum. This simplifies in particular the longitudinal part $\left(k_{+}^{\prime} k_{-}^{\prime}\right)$ of the loop integral in the second term. It reduces to $\left(\beta=2 k_{-} / \sqrt{s}\right)$

$$
\int_{0}^{\left(z-\sqrt{z^{2}-1}\right)} \frac{d \beta}{\beta} \beta^{\omega}=\frac{1}{\omega} \quad\left\{\begin{array}{cc}
1, & z \sim 1 \\
\left(\frac{2}{z}\right)^{\omega}, & z \rightarrow \infty
\end{array}\right.
$$

$z$ is the cosine of the $t$-channel scattering angle. If $k^{2} \approx-|\kappa|^{2}$ and $k^{\prime 2} \approx-\left|\kappa^{\prime}\right|^{2}$ are of the same order then $z$ is of order 1 and the longitudinal integral yields just $\omega^{-1}$. This step is usually done without long discussion. However in our case the ranges of integration where the ratio of $|\kappa|$ and $\left|\kappa^{\prime}\right|$ is either large or small are essential. There $z$ is approximated by the ratio of the maximum to the minimum of $|\kappa|$ and $\left|\kappa^{\prime}\right|$ and the second case in (2.5) applies.

The equation Fig. 3 is written in the following form

$$
\begin{array}{rc}
\kappa^{*}(q-\kappa)\left[\omega-\alpha_{F}(\kappa)-\alpha_{F}(q-\kappa)\right] \quad \tilde{f}(\omega, \kappa, \bar{\kappa}, q) & =\delta^{(2)}(\kappa-\bar{\kappa}) \\
+\frac{g^{2} C_{2}}{(2 \pi)^{3}} \int d^{2} \kappa^{\prime} \frac{\kappa^{\prime *}\left(q-\kappa^{\prime}\right)+\kappa^{*}(q-\kappa)}{\left|\kappa-\kappa^{\prime}\right|^{2}} & \left(\frac{2|\kappa|\left|\kappa^{\prime}\right|}{\left(|\kappa|+\mid \kappa^{\prime}\right)^{2}}\right)^{\omega} \tilde{f}\left(\omega, \kappa^{\prime}, \bar{\kappa}, q\right)
\end{array}
$$

We project on the colour singlet state in the $t$-channel. The $S U(N)$ gauge group generators in the vertices reduce to the factor $C_{2}=\left(N^{2}-1\right) / 2 N$ multiplying the kennel in (2.7). The second factor in the integrand takes the effect of the upper limit in (2.5) approximately into accout. $\omega$ can be considered as a small parameter and therefore this factor can be approximated in different ways. In the following form of the equation we choose an approximation most convenient for our purposes. It is reasonable to redefine the reggeon Green function $\tilde{f}$ by including the factor $\kappa^{*}(q-\kappa)$. We suppress the argument $\bar{\kappa}$ and write an arbitrary Born term.

$$
\begin{gathered}
\omega f(\kappa, q)=f_{0}(\omega, \kappa, q) \\
+\frac{g^{2} C_{2}}{(2 \pi)^{3}} \int \frac{d^{2} \kappa^{\prime}}{\left|\kappa-\kappa^{\prime}\right|^{2}}\left[\left(\frac{|\kappa|}{\left|\kappa^{\prime}\right|}\right)^{\omega}+\left(\frac{\left|\kappa^{\prime}\right|}{|\kappa|}\right)^{\omega} \frac{\kappa^{*}(q-\kappa)}{\kappa^{\prime *}\left(q-\kappa^{\prime}\right)}\right] f\left(\omega, \kappa^{\prime}, q\right) \\
-\frac{g^{2} C_{2}}{(2 \pi)^{3}} \int \frac{d^{2} \kappa^{\prime}}{\left|\kappa-\kappa^{\prime}\right|^{2}}\left(\frac{\kappa^{*}}{\kappa^{\prime *}}+\frac{q-\kappa}{q-\kappa^{\prime}}\right) \quad f(\omega, \kappa, q)
\end{gathered}
$$


The infrared divergences in the integrals at $\kappa=\kappa^{\prime}$ may be regularized by going to $2+2 \epsilon$ dimensions. Actually the divergencies cancel in the sum of the two integrals in (2.8). The first integrand becomes large also in the regions $\left|\kappa^{\prime}\right| \gg|\kappa| \gg|q|$ and $|\kappa| \gg\left|\kappa^{\prime}\right| \gg|q|$. However the divergences are prevented by the $\omega$-dependent factors for $\mathcal{R} e \omega<0$.

\section{Eigenvalue spectrum and conformal symmetry}

At vanishing momentum transfer, $q=0$, we have rotation symmetry in $\kappa$-plane and the appropriate orthogonal basis for functions of $\kappa$ is

$$
\varphi_{n, \nu}(\kappa)=\left|\kappa^{2}\right|^{-1 / 2+i \nu}\left(\frac{\kappa^{*}}{|\kappa|}\right)^{n},
$$

where $n$ runs over all integers and $\nu$ runs over the real axis. We prefer to define the scalar product for our functions $f(\omega, \kappa)$ with a weight, corresponding to the product of two fermion propagators. Therefore we decompose with respect to

$$
f_{n, \nu}=|\kappa|^{1 / 2} \varphi_{n, \nu}(\kappa)
$$

With (3.2) we diagonalize the homogeneous equation and obtain the eigenvalue equation

$$
\omega=\frac{g^{2} C_{2}}{8 \pi^{2}} \Omega(\omega, \nu, n)
$$

where

$$
\begin{gathered}
\Omega(\omega, \nu, n)=4 \psi(1)- \\
\psi\left(-i \nu+\frac{|n|}{2}+\frac{\omega}{2}\right)-\psi\left(i \nu+\frac{|n|}{2}+\frac{\omega}{2}\right)-\psi\left(1-i \nu-\frac{|n|}{2}+\frac{\omega}{2}\right)-\psi\left(1+i \nu-\frac{|n|}{2}+\frac{\omega}{2}\right), \\
\psi(z)=\frac{d}{d z} \ln \Gamma(z) .
\end{gathered}
$$

As the solution of the inhomogeneous equation (2.7) we obtain the reggeon Green function at $q=0$.

$$
\tilde{f}(\omega, \kappa, \bar{\kappa}, 0)=\frac{1}{2 \pi^{2}} \int_{-\infty}^{\infty} d \nu \sum_{n=-\infty}^{\infty} \frac{\left|\kappa^{2}\right|^{-1+i \nu}\left(\frac{\kappa}{|\kappa|}\right)^{n}\left|\bar{\kappa}^{2}\right|^{-1-i \nu}\left(\frac{\bar{\kappa}}{|\bar{\kappa}|}\right)^{-n}}{\omega-\frac{g^{2} C_{2}}{8 \pi^{2}} \Omega(\omega, \nu, n)}
$$

Each term in the sum over $n$ has a square-root branch point in $\omega$, arising from pinching the integration path by two poles in $\nu$. The positions of the branch points are the solution of

$$
\omega_{n}=\frac{g^{2} C_{2}}{8 \pi^{2}} \quad \Omega\left(\omega_{n}, 0, n\right) .
$$

The dominant ones are at $n=0$,

$$
\omega_{0}=\left(\frac{g^{2} C_{2}}{2 \pi^{2}}\right)^{1 / 2}\left(1+\mathcal{O}\left(g^{2}\right)\right)
$$


and at $n= \pm 1$,

$$
\omega_{1}=\frac{g^{2} C_{2}}{\pi^{2}}\left(\ln 2-\frac{1}{2}\right) \quad\left(1+\mathcal{O}\left(g^{2}\right)\right) .
$$

All other singularities are less important, $\omega_{n}<0,|n|>1$. (3.7) reproduces the result of the double logarithmic approximation [13]. The double logarithmic contributions show up essentially at $n=0$ and lead only to small corrections at $n \neq 0$.

In the case of exchanging two reggeized gluons (perturbative pomeron) the properties of conformal symmetry and holomorphic factorization have been observed [2] [16]. For these properties to hold it is neceesary that the spectrum $\Omega(\omega, \nu, n)$ can be written as a sum of two terms, one being a function of the eigenvalues $m(1-m)$ of the holomorphic and the other of the eigenvalues $\tilde{m}(1-\tilde{m})$ of the antiholomorphic conformal Casimir operator, where

$$
m=\frac{1}{2}+i \nu+\frac{n}{2}, \quad \tilde{m}=\frac{1}{2}+i \nu-\frac{n}{2} .
$$

$n$ enters (3.4) as $|n|$. A property of $\psi(z)$ allows to rewrite the eigenvalue function as a combination of $\psi$-functions avoiding absolute values.

$$
\begin{gathered}
\Omega(\omega, \nu, n)=4 \psi(1)- \\
\frac{1}{2}\{\psi(m+\Delta)+\psi(1-m+\Delta)+\psi(m-\Delta)+\psi(1-m-\Delta) \\
+\psi(\tilde{m}+\Delta)+\psi(1-\tilde{m}+\Delta)+\psi(\tilde{m}-\Delta)+\psi(1-\tilde{m}-\Delta)\},
\end{gathered}
$$

where $\Delta=(1-\omega) / 2$. Further we have

$$
2 \psi(1)-\psi(m+\Delta)-\psi(1-m-\Delta)=\chi_{\Delta}(m(1-m)),
$$

with the notation

$$
\chi_{\Delta}(x)=\sum_{\ell=0}^{\infty}\left(\frac{2(\ell+\Delta)+1}{(\ell+\Delta)(\ell+\Delta+1)+x}-\frac{2}{\ell+1}\right) .
$$

This allows to write the eigenvalue function as

$$
\begin{gathered}
\Omega(\omega, \nu, n)= \\
\frac{1}{2}\left(\chi_{\Delta}(m(1-m))+\chi_{-\Delta}(m(1-m))+\chi_{\Delta}(\tilde{m}(1-\tilde{m}))+\chi_{-\Delta}(\tilde{m}(1-\tilde{m})) .\right.
\end{gathered}
$$

We have shown that the spectrum has the desired properties. This means, that the equation (2.8) can be represented up to non-leading terms in a conformally symmetric form allowing holomorphic factorization.

\section{Representation in impact parameter space}

Instead of finding an integral representation in impact parameters as in [2] we shall work with operators as in [8]. The momenta in (2.7) translate into derivatives with respect to the coordinates $x_{1}, x_{2}$

$$
\kappa^{*} \rightarrow \partial_{1}, \quad q-\kappa \rightarrow \partial_{2}^{*},
$$


the transverse momentom factor in the gluon propagator translates as

$$
\frac{1}{\mid \kappa-\kappa^{\left.\prime\right|^{2}}} \rightarrow \quad-\ln \left|x_{12}^{2}\right|
$$

where $x_{12}=x_{1}-x_{2}$, and the fermion trajectory

$$
\alpha(\kappa) \rightarrow-\ln \left|\partial^{2}\right|+2 \psi(1)
$$

(4.2) and (4.3) can be understood in dimensional regularization, where the pole term $\sim \epsilon^{-1}$ is omitted in both expressions. Indeed the pole terms cancel in the operator below, because there are no infrared divergences in (2.7).

We write the equation (2.7) as

$$
\omega \tilde{f}=\tilde{f}_{0}+\frac{g^{2} C_{2}}{8 \pi^{2}} \mathcal{H}_{F \bar{F}} \tilde{f}
$$

In the impact parameter representation the partial wave depends on four points in the impact parameter space, $\tilde{f}=\tilde{f}\left(\omega, x_{1}, x_{2} ; \bar{x}_{1}, \bar{x}_{2}\right)$. The operator acts on $x_{1}, x_{2}$ only. It can be read off directla from (2.7) using (4.1) - (4.3).

$$
\begin{gathered}
\mathcal{H}_{F \bar{F}}^{(\omega)}=-\left(\partial_{1} \partial_{2}^{*}\right)^{-1}\left(\left(\partial_{1} \partial_{2}^{*}\right)^{\omega / 2} \ln \left|x_{12}^{2}\right|\left(\partial_{1} \partial_{2}^{*}\right)^{1-\omega / 2}+\left(\partial_{1}^{*} \partial_{2}\right)^{-\omega / 2} \partial_{1} \partial_{2}^{*} \ln \left|x_{12}^{2}\right| \quad\left(\partial_{1}^{*} \partial_{2}\right)^{\omega / 2}\right) \\
-\ln \left|\partial_{1}^{2}\right|-\ln \left|\partial_{2}^{2}\right|+4 \psi(1)
\end{gathered}
$$

The effect of the upper limit of the integration is included in a way similar to (2.8). $\mathcal{H}_{F \bar{F}}$ allows holomorphic factorization, because it decomposes into the sum $H_{F}+\tilde{H}_{F}$, where $H_{F}$ acts only on the holomorphic coordinates $x_{1}, x_{2}$ and $\tilde{H}_{F}$ only on $x_{1}^{*}, x_{2}^{*}$.

$$
\begin{aligned}
& H_{F}^{(\omega)}=\quad-\left(\partial_{1}\right)^{-1+\omega / 2} \ln x_{12}\left(\partial_{1}\right)^{1-\omega / 2}-\ln \partial_{1} \\
& -\left(\partial_{2}\right)^{-\omega / 2} \ln x_{12}\left(\partial_{2}\right)^{\omega / 2}-\ln \partial_{2}+2 \psi(1)
\end{aligned}
$$

$\tilde{H}_{F}^{(\omega)}$ is obtained from (4.6) by complex conjugation and interchanging the indices 1 and 2 .

We present several forms for $H_{F}$ in the limiting case $\omega=0$. Mathematically this limit makes sense for the operator although in the corresponding equation one would loose the dominant double-logarithmic contributions. The following discussion is analogous to the one for the two-gluon exchange [9].

Applying the commutation relation to the first term in (4.6) (at $\omega=0$ ) we obtain

$$
H_{F}^{(0)}=-2 \ln x_{12}-\ln \partial_{1}-\ln \partial_{2}-\partial_{1}^{-1}\left(x_{12}\right)^{-1}+2 \psi(1),
$$

which can be written by an analogous application of the commutation relation as

$$
H_{F}^{(0)}=-2 \ln x_{12}-\ln \partial_{2}-x_{12} \quad \ln \partial_{1} \quad x_{12}^{-1}+2 \psi(1) .
$$

Using the operator relation

$$
\ln \left(x^{2} \partial\right)-\ln x=\partial^{-1} \quad \ln x \quad \partial-\ln \partial
$$


one obtains from (4.6)

$$
H_{F}^{(0)}=-\ln \left(x_{12}^{2} \partial_{1}\right)-\ln \partial_{2}+2 \psi(1)
$$

The last form is convenient for studying the behaviour under conformal transformations. In particular under inversions $\mathcal{I} H_{F}^{(0)}$ transforms in the following way.

$$
\mathcal{I} \quad H_{F}^{(0)} \quad \mathcal{I}=x_{2} H_{f}^{(0)} x_{2}^{-1}
$$

The forms (4.6) and (4.8) imply that the transposed operator can be obtained from $H_{F}^{(0)}$ by two different similarity transformations,

$$
H_{F}^{(0) T}=\partial_{1} H_{F}^{(0)} \partial_{1}^{-1}=P_{12} x_{12}^{-1} H_{F}^{(0)} x_{12} P_{12}
$$

Here $P_{12}$ denotes the operator permuting $x_{1}$ and $x_{2}$. From (4.12) we understand, that the following operator commutes with $H_{F}^{(0)}$.

$$
A_{F}=P_{12} x_{12} \partial_{1}, \quad\left[A_{F}, H_{F}^{(0)}\right]=0 .
$$

The operator relation (4.9) can be derived by observing that

$$
\begin{aligned}
x^{2} \partial & =\Gamma(x \partial) x(\Gamma(x \partial))^{-1} \\
\partial & =(\Gamma(x \partial+1))^{-1} x^{-1} \Gamma(x \partial+1) .
\end{aligned}
$$

The operators in the similarity transformation are actually determined only up to periodic functions with the period 1 of the same argument. This ambiguity matters, if we apply (4.14) to logarithms.

(4.14) implies that both sides of (4.9) are equal to $\psi(x \partial)$. It also allows to write (4-10) as a sum of $\psi$-functions.

$$
H_{F}^{(0)}=-\frac{1}{2}\left(\psi\left(D_{1}+1\right)+\psi\left(-D_{1}\right)+\psi\left(D_{2}+1\right)+\psi\left(-D_{2}\right)\right)+2 \psi(1)
$$

We have used the notation $D_{1}=x_{12} \partial_{1}, D_{2}=x_{12} \partial_{2}$. There is an uncertainty in the last form of the operator due to the periodic function ambiguity in (4.14).

The operator $H_{F}^{\omega}$ at $\omega \neq 0$ is more complicated. As it stands, it does not have the symmetry properties (4.11) and (4.13). On the other hand we know, that up to nonleading terms it approximates an operator with nice symmetry properties.

Applying (4.14) and going the analogous steps which led to (4.15) we obtain, that up to non-leading contributions

$$
\begin{gathered}
H_{F}^{(0)}=-\frac{1}{2} \partial_{1}^{-\omega / 2}\left(\psi\left(D_{1}+1\right)+\psi\left(-D_{1}\right)\right) \partial_{1}^{\omega / 2}-\frac{1}{2} \partial_{2}^{-\omega / 2}\left(\psi\left(D_{2}+1\right)+\psi\left(-D_{2}\right)\right) \partial_{2}^{\omega / 2}+2 \psi(1) \\
=-\frac{1}{2}\left(\psi\left(1+D_{1}-\frac{\omega}{2}\right)+\psi\left(1+D_{2}-\frac{\omega}{2}\right)\right) \\
-\frac{1}{2}\left(\psi\left(-D_{1}+\frac{\omega}{2}\right)+\psi\left(-D_{2}+\frac{\omega}{2}\right)\right)+2 \psi(1)
\end{gathered}
$$




\section{$5 \quad$ Using conformal symmetry}

Now we consider the diagonalization of ther equation (4.4) by means of conformal symmetry. We have seen that the equation with $H_{F}^{(0)}$ is symmetric and we know that it can be made symmetric by non-leading corrections also with $H_{F}^{(\omega)}$. The functions $\tilde{f}$ can be expanded in conformal 3-point functions,

$$
\begin{array}{r}
E\left(x_{10}, x_{20}\right)=\left\langle\phi^{\left(\Delta_{1}, \tilde{\Delta}_{1}\right)}\left(x_{1}\right) \phi^{\left(\Delta_{1}, \tilde{\Delta}_{1}\right)}\left(x_{2}\right) \mathcal{O}^{(m, \tilde{m})}\left(x_{0}\right)\right\rangle \\
=\left(\frac{x_{12}}{x_{10} x_{20}}\right)^{m-\Delta_{1}-\Delta_{2}} x_{10}^{-2 \Delta_{1}} x_{20}^{-2 \Delta_{2}} \cdot\left(\frac{x_{12}^{*}}{x_{10}^{*} x_{20}^{*}}\right)^{\tilde{m}-\tilde{\Delta}_{1}-\tilde{\Delta}_{2}} x_{10}^{*-2 \tilde{\Delta}_{1}} x_{20}^{*-2 \tilde{\Delta}_{2}}, \\
\Delta_{1 / 2}=\frac{1}{2}\left(\delta_{1 / 2}+s_{1 / 2}\right), \quad \tilde{\Delta}_{1 / 2}=\frac{1}{2}\left(\delta_{1 / 2}-s_{1 / 2}\right), \\
m=\frac{1}{2}(\delta+n), \quad \tilde{m}=\frac{1}{2}(\delta-n) .
\end{array}
$$

$\delta_{1 / 2}, \delta$ are the scaling dimensions and $s_{1 / 2}, n$ are the conformal spins of the operators in the points $x_{1 / 2}, x_{0}$. The behaviour of $H_{F}^{(0)}$ under inversion (4.11) shows that $\tilde{f}$ transforms non-trivially. We arrive at the conclusion that the operators in $x_{1 / 2}$ corresponding to the exchanged fermions carry conformal weights

$$
\Delta_{1}=0, \quad \Delta_{2}=\frac{1}{2}, \quad \tilde{\Delta}_{1}=\frac{1}{2}, \quad \tilde{\Delta}_{2}=0 .
$$

The 6 generators of the conformal transformations in the corresponding representation on functions of $x_{1}, x_{2}$ have the form

$$
\begin{gathered}
M_{12}^{0}=x_{1} \partial_{1}+x_{2} \partial_{2}+\Delta_{1}+\Delta_{2}, \quad M_{12}^{-}=\partial_{1}+\partial_{2}, \\
M_{12}^{+}=x_{1}^{2} \partial_{1}+2 \Delta_{1} x_{1}+x_{2}^{2} \partial_{2}+2 \Delta_{2} x_{2},
\end{gathered}
$$

with obvious modification for the anti-holomorphic generators $\tilde{M}_{12}^{ \pm}, \tilde{M}_{12}^{0}$. They obey the commutation relations

$$
\left[M_{12}^{0}, M_{12}^{ \pm}\right]= \pm M_{12}^{ \pm}, \quad\left[M_{12}^{+}, M_{12}^{-}\right]=-2 M_{12}^{0} .
$$

We need the explicite form of the Casimir operator

$$
C_{\Delta_{1}, \Delta_{2}}=-\left(M_{12}^{0}\right)^{2}+\frac{1}{2}\left(M_{12}^{+} M_{12}^{-}+M_{12}^{-} M_{12}^{+}\right)
$$

for our case (5.2)

$$
C_{0, \frac{1}{2}}=x_{12}^{2} \partial_{1} \partial_{2}-x_{12} \partial_{1}+\frac{1}{4} .
$$

We notice a remarkable property, which may be related to supersymmetry,

$$
A_{F}^{2}=-C_{0, \frac{1}{2}}+\frac{1}{4} \text {. }
$$


Therefore the commutativity (4.13) of $H_{F}^{(0)}$ with $A_{F}$ is just another way to express its conformal symmetry.

The properties of orthogonality and completeness of the conformal 3-point functions discussed in [2] [19] for $\Delta_{1}=\Delta_{2}=0$ hold also in the general case. We shall not write these relations and the expansion of the solution of (2.7), (4.4), the reggeon Green function, in conformal 3-point functions here, because the form is like in [2] [19]. We have the twofold overcomplete orthogonal set (5.1) of functions of $x_{1}, x_{2}$ parametrized by $\delta=$ $\frac{1}{2}+i \nu,-\infty<\nu<\infty$, by $n$ running over all integers and by the position $x_{0}$ running over the whole complex plane. This becomes evident, if we transform to the $\rho q$ representation, where $\rho=x_{12}$ and the momentum transfer $q$ is the Fourier conjugate to $R=\left(x_{10}+x_{20}\right) / 2$. In the asymptotics $\rho q \ll 1$ we come close to the rotation symmetric situation (3.1) and obtain for the conformal 3-point functions the simple approximation

$$
\begin{aligned}
& \tilde{E}_{\delta_{1}, \delta_{2}, s_{1}, s_{2}}^{n, \nu}=|\rho|^{1-\delta_{1}-\delta_{2}}\left(\frac{\rho}{\rho^{*}}\right)^{\frac{s_{1}+s_{2}}{2}} r_{n, \nu} . \\
& {\left[|\rho|^{-2 i \nu}\left(\frac{\rho}{\rho^{*}}\right)^{\frac{n}{2}}+e^{i \delta(n, \nu)}\left|q^{2} \rho\right|^{2 i \nu}\left(\frac{q^{* 2} \rho}{q^{2} \rho^{*}}\right)^{-\frac{n}{2}}\right] \quad(1+\mathcal{O}(\rho q)) \text {. }}
\end{aligned}
$$

The constants $r_{n, \nu}$ and $\delta(n, \nu)$ are known. In the following we need the phase

$$
\begin{gathered}
e^{-\delta(n, \nu)}=2^{4 i \nu} \\
(2 i \nu+|n|) \Gamma^{2}(2 i \nu+|n|) \Gamma\left(-i \nu+\frac{|n|+1}{2}-\frac{\left|s_{1}-s_{2}\right|}{2}\right) \Gamma\left(i \nu+\frac{|n|+1}{2}+\frac{\left|s_{1}-s_{2}\right|}{2}\right) \\
\text { c.c. }
\end{gathered}
$$

The conformsymmetric operator $H_{F s y m}^{(\omega)}$, a non-symmetric approximation to which is given by (4.16), is a function of the Casimir operator $C_{0, \frac{1}{2}}(5.6)$. This holds in particular in the asymptotics $\rho q \ll 1$, where the approximation (4.16) is good. On the other hand in this asymptotics

$$
D_{1}+D_{2} \sim \rho q \ll 1
$$

We start from (4.16), use the formula

$$
\psi(1)-\psi(z)=\sum_{\ell=0}^{\infty}\left(\frac{1}{\ell+z}-\frac{1}{\ell+1}\right)
$$

and (5.6)

$$
C_{0, \frac{1}{2}}=D_{2} D_{1}+\frac{1}{4} .
$$

In the approximation (5.10) we have

$$
\begin{array}{r}
\frac{1}{\ell+1+D_{1}-\frac{\omega}{2}}+\frac{1}{\ell+1+D_{2}-\frac{\omega}{2}} \\
\frac{2 \ell+2-\omega}{\left(\ell+1-\frac{\omega}{2}\right)^{2}+D_{2} D_{1}}
\end{array}=
$$


In this way we obtain the expression of $H_{F s y m}^{(\omega)}$ in terms of the conformal Casimir operator.

$$
H_{F s y m}^{(\omega)}=\frac{1}{2}\left(\chi_{\Delta}\left(C_{0, \frac{1}{2}}\right)+\chi_{-\Delta}\left(C_{0, \frac{1}{2}}\right)\right)
$$

where the notation (3.12) has been used with $\Delta=(1-\omega) / 2$. Together with the analogous expression for $\tilde{H}_{F s y m}^{(\omega)}$ this confirms the result (3.13) about the eigenvalues spectrum.

\section{$6 \quad$ Regge trajectories at relatively large momentum transfer}

The expression of the reggeon Green function in terms of conformal 3-point functions obtained from (4.4) generalizes (3.5) to arbitrary momentum transfer $t=q^{2}$. It has a form analogous to (3.5) and the singularities in $\omega$ arise just in the same way. The perturbative Regge singularities do not move with $t$ in the leading logarithmic approximation with a fixed coupling $g$.

The approximation of a fixed coupling is reasonable in the channels without double logarithms, if $t$ and $\kappa^{2}$ are of the same order. Then this scale determines the coupling, because essentially all momenta sqared are of this order. In channels dominated by doublelogarithmic contributions the region of applicability of the fixed coupling approximation is more restricted. The transverse momentum integrals may get essential contributions from $\kappa^{\prime 2} \gg \kappa^{2} \sim t$. However for $\omega$ not small, $\omega \gg g^{2}$, such contributions are suppressed. Under this condition on $\omega$ also in this case the fixed coupling approximation can be justified.

Actually the eqation has been derived in the approximation of small $\omega$. At small coupling there is an intermediate region, where $\omega$ is small enough for this approximation and still provides enough suppression of large transverse momenta. Indeed, as we have seen, the leading singularity in the double logarithmic channel (5.7) is at $\omega_{0} \sim g \gg g^{2}$.

In both cases the fixed coupling approximation is not true anymore, if $\kappa$ becomes large (or $\rho$ small ), which is the deep- inelastic region. There the dependence on $\rho$ ( or on $\kappa$ ) is determined by the renormalization group equation. The equation (2.7), (4.4) has to be modified to become compatible with the renormalization group.

We write (4.4) in the $\rho q$ representation approximated for $\rho q \ll 1$.

$$
\begin{array}{r}
\omega f(\rho)=\frac{g^{2} C_{2}}{8 \pi^{2}} \quad \Omega(\omega, \hat{\nu}, n) \quad f(\rho), \\
\hat{\nu}=i \frac{\partial}{\partial \ln \left(|\rho|^{2} \Lambda^{2}\right)}
\end{array}
$$

We restrict ourselves to the channels with conformal spin $n=0, \pm 1$, where the leading singularities (3.7), (3.8) arise. The solution have the form (5.8) with the conformal weights (5.2). Inverting $\Omega$ as a function of $\nu(6.1)$ can be written as

$$
|\rho|^{2} \frac{\partial}{\partial|\rho|^{2}} \quad f(\rho)=\nu\left(\frac{g^{2}}{4 \pi}, \omega, n\right) \quad f(\rho)
$$


The renormalization group leads to replacing

$$
\frac{g^{2}}{4 \pi} \rightarrow \alpha_{S}(1 / \rho)=\frac{-b}{\ln \left(|\rho|^{2} \Lambda^{2}\right)}
$$

in the scaling dimension $\nu$ in (6.2). $b$ is the leading coefficient in the Gell-Mann - Low function.

Instead of studying (6.2) with the replacement (6.3) we solve (6.1) with the replacement (6.3). The asymptotics of the solutions at $\rho \rightarrow 0$ is the same in both cases and is given by the saddle point approximation of the integral

$$
f(\rho)=\int_{-\infty}^{\infty} d \nu \exp \left[-i \nu \ln \left(|\rho|^{2} \Lambda^{2}\right)+\frac{i b C_{2}}{2 \pi \omega} \int_{0}^{\nu} \Omega\left(\omega, \nu^{\prime}, n\right) d \nu^{\prime}\right]
$$

The saddle point equation coincides with (3.3) after the replacement (6.3).

In the region where $\rho q \ll 1$ but still

$$
\alpha_{S}\left(q^{2}\right) \ln \left(|\rho q|^{-2}\right) \ll 1
$$

the solutions of the original equation (6.1) and the one modified by (6.3) should match. In particular the condition for the phases to match (modulo $\pi$ ) restricts the possible values of $\nu$ to a discrete set $\nu^{(r)}(\omega), \quad r$ integer.

$$
\begin{array}{r}
\nu^{(r)} \ln \left(|\rho|^{2} q^{2}\right)+\frac{1}{2} \delta\left(n, \nu^{(r)}\right)+\pi r= \\
-\frac{\pi}{4}+\nu^{(r)} \ln \left(|\rho|^{2} \Lambda^{2}\right)+\frac{b C_{2}}{2 \pi \omega} \int_{0}^{\nu^{(r)}} \Omega\left(\omega, \nu^{\prime}, n\right) d \nu^{\prime}
\end{array}
$$

In the solution obeying the condition of continuity between the perturbative Regge (fixed coupling) region and the deep inelastic region instead of the integral over $\nu$ like in (3.5) there is a sum over $r$ with the solutions of $(6.6) \nu^{(r)}$ inserted. The singularities in $\omega$ are now a series of poles at $\omega_{n}^{(r)}$, the solution of

$$
\omega_{n}^{(r)}=\alpha_{S}\left(q^{2}\right) \frac{C_{2}}{2 \pi} \quad \Omega\left(\omega_{n}^{(r)}, \nu_{n}^{(r)}, n\right) .
$$

We solve (6.6), (6.7) for $n=0, \pm 1$ within our approximation. For this we use the approximation of $\Omega(\omega, \nu, n)$ (3.4) for small $\omega, \nu$ disregarding terms beyond the second order in $\omega, \nu$ and also the value of the phase $\delta(n, \nu)$ at $\nu=0$.

At $n=0$ we obtain

$$
\omega_{0}^{(r)}=\left(\frac{2 C_{2} \alpha_{S}\left(q^{2}\right)}{\pi}\right)^{1 / 2}\left[1-\left(\frac{\alpha_{S}\left(q^{2}\right)}{C_{2}}\right)^{1 / 3} \pi\left(\frac{3}{8 b}\left(r+\frac{1}{4}\right)\right)^{2 / 3}\right]
$$

and at $n= \pm 1$

$$
\omega_{1}^{(r)}=\frac{2 \alpha_{S}\left(q^{2}\right) C_{2}}{\pi}(2 \ln 2-1)\left[1-\left(\alpha_{S}\left(q^{2}\right)\right)^{2 / 3}\left(\frac{21 \zeta(3)-4}{2 \ln 2-1}\right)^{1 / 3}\left(\frac{3 \pi}{2 b}\left(r+\frac{1}{4}\right)\right)^{2 / 3}\right]
$$


Applying this method the Regge trajectories were calculated for the channel $n=0$ earlier in [20]. The phase $\delta(n, \nu)$ was left undetermined there.

Only a discrete set of scaling dimensions $\nu^{(r)}$ allows the continuation of the solution from the perturbative Regge region to the deep inelatic region. Instead of the branch point we obtain an infinite series of Regge poles moving with $t=q^{2}$. In the region of relatively large $|t|$, where the perturbative leading logarithmic calculation gives a reasonable estimate, the $t$ dependence comes through the renormalized coupling.

\section{Discussion}

We have extended the methods of conformal symmetry in the Regge asymptotics to the flavour exchange channels of QCD. Here the leading contributions to the perturbative Regge asymptotics arise from the exchange of two reggeized fermions ( quark and antiquark of different flavour) and it is determined by double logarithmic contrbutions, if the fermions carry opposite helicities.

We have shown that the eigenvalue spectrum of the partial-wave equation obeys the necessary conditions for conformal symmetry and factorization. Therefore the equation can be written in a conformsymmetric form. We have obtained this symmetric form using the operator representation in impact parameter space. The equation is written in terms of an operator, which is a sum of a function of the holomorphic plus a function of the anti-holomorphic Casimir operators of the linear conformal tranformations.

We have obtained the leading perturbative Regge singularities in the flavour exchange channels. The singularity in the channel with conformal spin $n=0$ arises from doublelogarithmic contributions and it dominates the channels with higher conformal spin. The result for the position of this branch point in the complex angular momentum plane coincides with the one obtained in the double-logarithmic approximation. This means, that in the leading $\ln s$ approximation there are no essential corrections to the double-

logarithmic result. As a practical consequence our equation ( $\ln \frac{1}{x}$ approximation ) and the LAP equation [22] $\left(\ln Q^{2}\right.$ approximation ) predict the same low $x$ asymtotics for the non-singlet structure functions.

We are able to calculate the singularities also in channels of other conformal spins $n$. They are determined essentially by single-logarithmic contributions. The case of $n= \pm 1$ is of interest because the branch point is located at positive angular momentum. This contribution is a correction to the one of $n=0$. There may be situations where the contribution of $n=0$ is suppressed due to the impact factors, i.e. the corresponding Regge singularity may have a small coupling to the scattering particles.

We have discussed the conditions of validity of the fixed coupling calculations and the necessary modifications when going towards the deep inelastic region. As a result the fixed branch points are converted into series of moving Regge poles. We have calculated the Regge trajectories in the channels $n=0$ and $n= \pm 1$ at relatively large momentum transfer. Earlier we observed the appearance of Regge poles by including the running coupling into the simple non-linear partial wave equation obtained in the double logarith- 
mic approximation [21]. It remains to be analyzed, how the two approaches ( for $n=0$ dominated by double logarithms) compare in detail.

The results about the trajectories give no information on the slope, the intercept of the meson trajectories or on the meson masses. However it becomes clear, that the Regge singularity structure in the meson channels as well as in the vacuum channel is more complicated in QCD than assumed usually in phenomenology. Any model of nonperturbative effects resulting in a definite modification of the equation in the region of small transverse momenta will produce by an analogous procedure predictions on the intercept and on the slope.

The operator representation in impact parameter space, which was used first in [8] [9] for the gluon exchange, has been worked out for the application to the flavour exchange channels. The conformal method has been extended to the case where the exchanged reggeons are attributed non-vanishing conformal spin and to the treatment of doublelogarithmic contributions. Now all two-reggeon interaction operators, which appear in the partial-wave equation in the general case of multiple exchange of reggeized quarks and gluons, can be treated in the same way [23]. The results about the integrability of the equation for an arbitrary number of exchanged gluons in the large $N$ approximation [10] generalize to the exchange of gluons and fermions. 


\section{Acknowledgements}

The author is grateful to L.N. Lipatov and L. Szymanowski for discussions.

\section{References}

[1] V.S. Fadin,E.A. Kuraev and L.N. Lipatov, Phys. Lett. 60B(1975)50; ZhETF 71(1976)840; ibid 72(1977)377

Y.Y. Balitski and L.N. Lipatov, Sov. J. Nucl. Phys. 28(1978) 882

[2] L.N. Lipatov, ZhETF 90 (1986), 536

[3] Proceedings of the Zeuthen workshop 1992, Nucl. Phys. B (Proc. Supll.) 29A (1992)

[4] A.H. Mueller and H. Navelet, Nucl. Phys. B282 (1987) 727

A.H. Mueller and W.-K. Tang, Phys. Lett. 284B (1992) 123

[5] J. Bartels, Nucl. Phys. B(Proc. Suppl.)29A(1992)44; Phys. Lett. 298B (1993) 204

[6] L.N. Lipatov, Nucl. Phys. B365(1991)614

[7] R. Kirschner, L.N. Lipatov and L. Szymanowski, "Effective action for multi-Regge processes in QCD", hep-th 9402010, Siegen Univ. preprint (1994); "Symmetry properties of the eff. action ...", hep-th 9403082, to appear as DESY preprint

[8] L.N. Lipatov, Phys. Lett 251B (1990) 284

[9] L.N. Lipatov, Phys. Lett. 309B (1993) 393

[10] L.N. Lipatov, "High-energy asymptotics of multi-colour QCD and exactly solvable lattice models", Padova preprint DFPD/93/TH/70 (1993)

[11] S.J. Brodsky, W.-K. Tang and C.B. Thorn, "The reggeon trajectory in exclusive and inclusive large momentum transfer reaction", preprint SLAC Pub 6227 (1993);

W.-K. Tang, "High-energy quark antiquark elastic scattering with meson exchange", preprint SLAC Pub 6024 (1993)

[12] R. Kirschner, Yadern. Fiz. 34 (1981) 446;

Phys. Lett.98B (1981) 451

[13] R. Kirschner and L.N. Lipatov, Nucl. Phys. B213 (1983) 521

[14] J. Kwiecinski, Phys. Rev. D26 (1982) 3293 
[15] R. Kirschner, Zeitschr. f. Phys. C31 (1986) 135

[16] L.N. Lipatov in "Perturbative QCD" A.H. Mueller ed., World Scientific 1989

[17] P. Gauron, L.N. Lipatov and B. Nicolescu, Phys. Lett. B260(1991)407; ibid B304(1993)334

[18] R. Kirschner, Zeitschr. f. Phys. C55 (1992) 289

[19] R. Kirschner and L.N. Lipatov, Zeitschr. f. Phys. C45 (1990) 477

[20] M. McGuigan and C.B. Thorn, Phys. Rev. Lett. 69 (1992) 1312

[21] R. Kirschner, Physica Scripta 28 (1983) 521

[22] V.N. Gribov and L.N. Lipatov, Sov. J. Nucl. Phys. 15(1972)438;

L.N. Lipatov, Sov. J. Nucl. Phys. 20(1975)94;

G. Altarelli and G. Parisi, Nucl. Phys. B26(1977)298

[23] R. Kirschner, "Operators of two-reggeon interaction in QCD", in preparation 


\section{Figure captions}

Fig. 1 Effective graphical rules. a) effective vertex of gluon emission, b) propagator of the exchanged fermion, c) propagator of the s-channel gluon.

Fig. 2 Amplitude high-energy scattering. The blobs denoted by $\Phi$ stand for the impact factors and the blob $f$ stands for the reggeon Green function.

$p_{A}$ and $p_{B}$ are the momenta of incomimg particles.

Fig. 3 Equation for the two-reggeon Green function. 


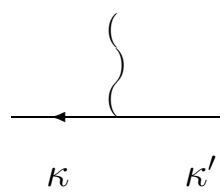

a

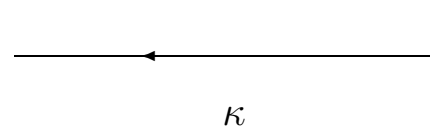

b $\left\{k-k^{\prime}\right.$

c

Fig.1

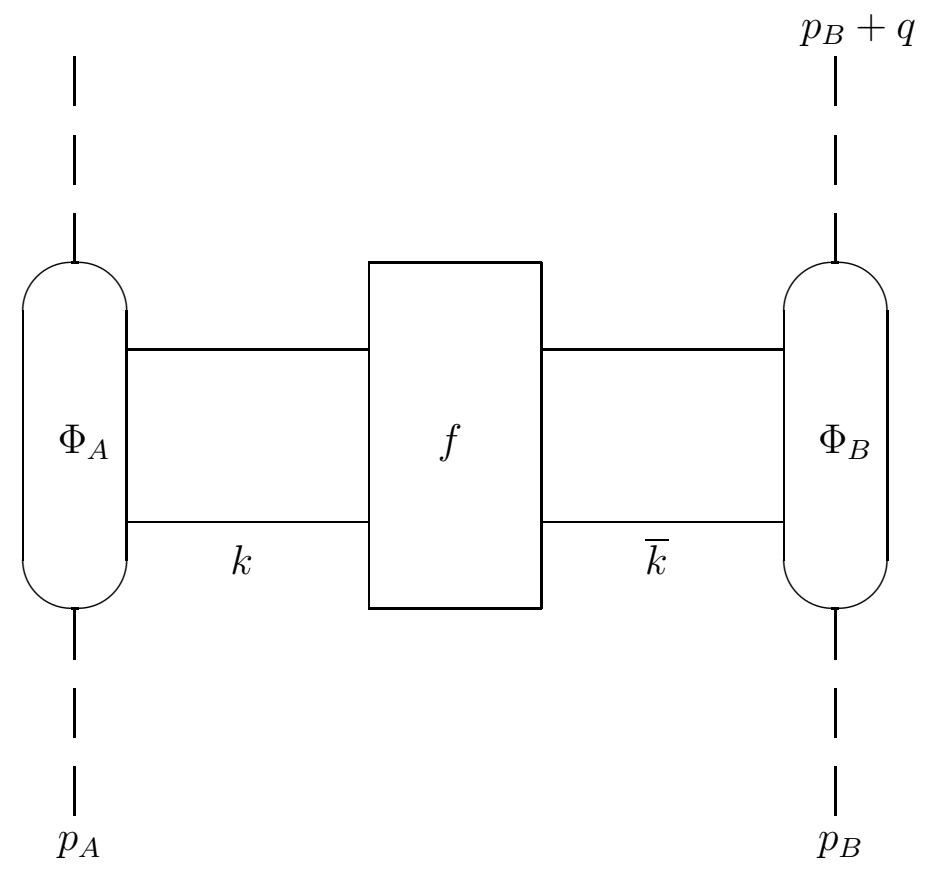

Fig. 2

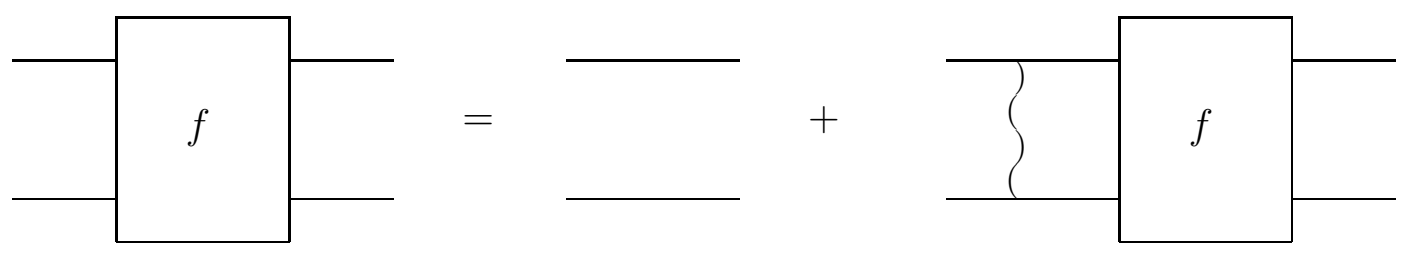

